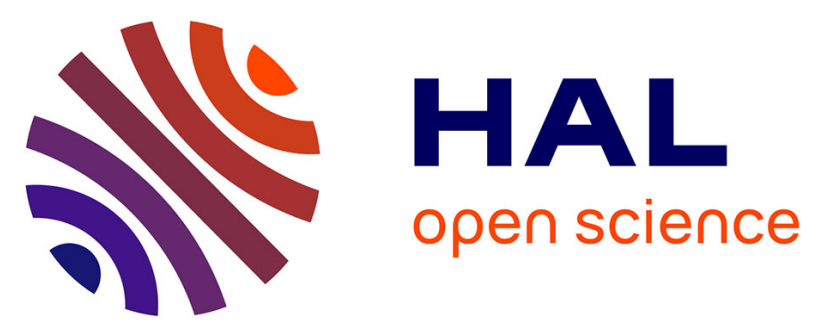

\title{
Germanane MOSFET for Subdeca Nanometer High-Performance Technology Nodes
}

Madhuchhanda Brahma, Marc Bescond, Demetrio Logoteta, Ram Krishna Ghosh, Santanu Mahapatra

\section{- To cite this version:}

Madhuchhanda Brahma, Marc Bescond, Demetrio Logoteta, Ram Krishna Ghosh, Santanu Mahapatra. Germanane MOSFET for Subdeca Nanometer High-Performance Technology Nodes. IEEE Transactions on Electron Devices, 2018, 65 (3), pp.1198-1204. 10.1109/TED.2017.2788463 . hal02331946

\section{HAL Id: hal-02331946 \\ https://hal.science/hal-02331946}

Submitted on 14 Jan 2022

HAL is a multi-disciplinary open access archive for the deposit and dissemination of scientific research documents, whether they are published or not. The documents may come from teaching and research institutions in France or abroad, or from public or private research centers.
L'archive ouverte pluridisciplinaire HAL, est destinée au dépôt et à la diffusion de documents scientifiques de niveau recherche, publiés ou non, émanant des établissements d'enseignement et de recherche français ou étrangers, des laboratoires publics ou privés. 


\title{
Germanane MOSFET for sub-Deca Nanometer High Performance Technology nodes
}

\author{
Madhuchhanda Brahma, Marc Bescond, Demetrio Logoteta, Ram Krishna Ghosh and Santanu Mahapatra
}

\begin{abstract}
Ballistic transport in monolayer Germanane metal oxide field effect transistors (MOSFETs) are investigated for high performance(HP) applications. Characteristics of both $n$ - and $\mathbf{p -}$ type transistors having channel lengths of $7 \mathrm{~nm}, 5 \mathrm{~nm}$ and $3 \mathrm{~nm}$ are studied and compared against the International Technology Roadmap for Semiconductor (ITRS) target of 2028. For this purpose we conceive single band effective mass Hamiltonian and double-band k.p Hamiltonian for $\mathbf{n}$ - and p-type channels respectively. The quantum transport model is based on the NonEquillibrium Greens Function (NEGF) formalism self consistently coupled with Poisson equation. The ON current $\left(I_{\mathrm{ON}}\right)$ in $3 \mathrm{~nm}$ channel $\mathbf{n}$ - and p-MOSFETs, for a fixed OFF current $I_{\text {OFF }}=100 \mathrm{nA} / \mu \mathrm{m}$, are found to be $\sim 890 \mu \mathrm{A} / \mu \mathrm{m}$ and $700 \mu \mathrm{A} / \mu \mathrm{m}$ respectively, which is indeed remarkable. We also observe that with the increase of channel lengths, the p-MOSFET starts to outperform the n-MOSFET in terms of $I_{\mathrm{ON}}$ requirements as the direct source-to-drain tunneling gets suppressed. Other performance metrics like total gate capacitance, intrinsic switching delay and switching energy have also been calculated and found to be comparable to the ITRS 2028 HP technology requirements.
\end{abstract}

Index Terms-Germanane, 2D materials, ballistic transport, ITRS, Non-Equillibrium Greens Function (NEGF) formalism.

\section{INTRODUCTION}

$\mathbf{I}$ $\mathrm{N}$ recent times, atomically thin two dimensional (2D) materials are being extensively explored as an alternative channel material for transistors in an aspiration of CMOS technology growth beyond the Moores law. Most of such materials e.g. transition metal di-chalcogenides or TMD $\left(\mathrm{MoS}_{2}\right.$, $\mathrm{WS}_{2}, \mathrm{MoSe}_{2}, \mathrm{WSe}_{2}, \mathrm{MoTe}_{2}$ ), Phosphorene, Graphene, hBN etc. [1]-[8] are naturally available in layered form, where two-dimensional platelets are weakly bonded by vander Waals force to form three-dimensional crystals. In an alternate route, recent efforts are also observed to extract 2D analogues from conventional bulk semiconductors like $\mathrm{Si}, \mathrm{Ge}, \mathrm{GaN}$ etc. [9]-[14]. Among these, hydrogenated monolayer of Germanium namely Germanane has attracted much attention. Unlike

This work was supported by Science and Engineering Research Board (SERB), Department of Science and Technology (DST), Government of India, under Grant SB/S3/EECE/0209/2015, Robert Bosch Cyber Physical System, Indian Institute of Science (IISc) Bangalore, under grant no: RBC00011 and Raman-Charpak Fellowship, Centre Franco-Indien pour la Promotion de la Recherche Avancée (CEFIPRA) under grant no. RCFIN-133. M. Brahma is with the Centre for Nano Science and Engineering (CeNSE) and S. Mahapatra is with Department of Electronic Systems Engineering (DESE), Indian Institute of Science (IISc) Bangalore, Bangalore 560012, India. R. K. Ghosh is with University of Notre Dame, IN 46556, USA. Marc Bescond and D.Logoteta are with IM2NP, CNRS, 3453 Marseille Cedex 13, France. email: madhu.brahma@gmail.com, marc.bescond@im2np.fr, demetrio.logoteta@im2np.fr, rghosh1@nd.edu, santanu@cedt.iisc.ernet.in
TMDs, which show higher band gap and higher effective mass, Germanane inherits a very interesting property of having low effective mass along with a relatively high band gap [14] in the group of 2D materials. From recent first principle based assessment [18], it was further concluded that chair morphology of Germanane has better potential for conventional and tunnel FET applications than its boat morphology. Stable structure of Germanane has been reported for the first time in 2013 [14], and later used for fabrication of Schottky diodes and FETs [15]-[17].

Since high performance switching devices demand channel materials with low effective mass to ensure high ON current, Germanane stands a very good chance in this respect. It is therefore important to theoretically estimate the performance limit of Germanane channel MOSFETs. However, previous studies in this context are either based on semi-classical approach [19] or limited only to the n-MOSFET [20]. In this work we focus on NEGF based ballistic transport in both n- and p-type Germanane chair FETs with channel lengths less than $10 \mathrm{~nm}$ to examine if such short Germanane FETs are able to meet the ITRS 2028 HP limit [21]. In section II of our paper, we describe the methodology used to simulate the transfer characteristics while in section III we present and analyse the results. From the transfer characteristics we find that the ON-state currents even in ultra-scaled Germanane nand p-MOSFETs of $3 \mathrm{~nm}$ show remarkably high values , $890 \mu \mathrm{A} / \mu \mathrm{m}$ and $\sim 700 \mu \mathrm{A} / \mu \mathrm{m}$ respectively. Both these values are comparable to the ITRS projected $2028 \mathrm{HP} I_{\mathrm{ON}}$ value of $900 \mu \mathrm{A} / \mu \mathrm{m}$. Finally, we have bench-marked the dynamic performance metrics of these sub-deca nanometer Germanane HP FETs with respect to the other state of the art 2D materials as well as the ITRS projection limits.

\section{Methodology}

In consideration with the ITRS-predicted physical gate length, effective oxide thickness, and power supply voltage for the 2028 HP devices, we conceive the device as shown in Fig. 1 with a channel length equal to the gate length and varying from $7 \mathrm{~nm}, 5 \mathrm{~nm}$ to $3 \mathrm{~nm}$, along with an effective oxide thickness(EOT) of $0.47 \mathrm{~nm}$, and a supply bias of $V_{\mathrm{D}}=$ $0.6 \mathrm{~V}$. The source(drain) lengths $L_{\mathrm{S}}\left(L_{\mathrm{D}}\right)$ are kept to be $30 \mathrm{~nm}$ each. 


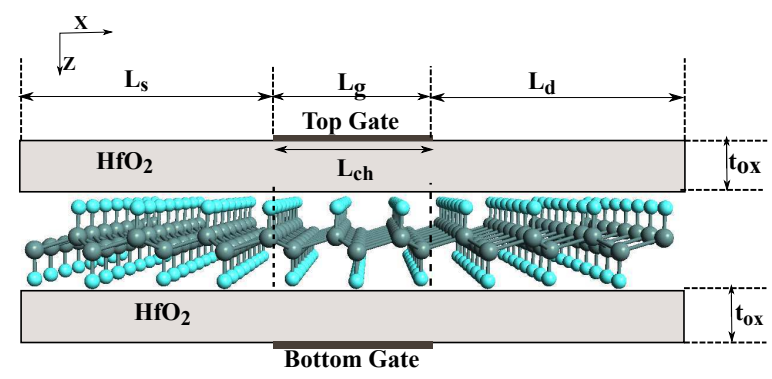

Fig. 1: Cross section of the device structure. $t_{\mathrm{ox}}=3 \mathrm{~nm}$. $L_{\mathrm{S}}$ $\left(L_{\mathrm{D}}\right)=30 \mathrm{~nm}$ in simulation. The length of the intrinsic channel is kept equal to the length of gate $L_{\mathrm{G}}$.

Moreover, the gate work-function is also adjusted such that the OFF-state current $\left(I_{\mathrm{OFF}}\right)$ at zero gate bias can be fixed at $100 \mathrm{nA} / \mu \mathrm{m}$. Monolayer Germanane chair has isotropic conduction band and valence bands (light hole and heavy hole) [18]. Therefore, for $\mathrm{n}$ type MOSFET we have chosen a single band effective mass Hamiltonian with electron effective mass of $0.071 m_{\circ}$. For the $\mathrm{p}$-type MOSFET, we developed a twoband k.p Hamiltonian based on the approach described in [22] for its analogous Graphene counterpart and given as

$$
H(\hat{k}) \equiv\left[\begin{array}{cc}
\frac{\gamma_{1}}{2}\left(\hat{k_{x}}+{\hat{k_{y}}}^{2}\right) & \frac{\gamma_{2}}{2}\left(\hat{k_{x}}+i \hat{k_{y}}\right)^{2} \\
\frac{\gamma_{1}}{2}\left(\hat{k_{x}}-i \hat{k_{y}}\right)^{2} & \frac{\gamma_{1}}{2}\left(\hat{k_{x}}+\hat{k}_{y}^{2}\right)
\end{array}\right],
$$

with the constants

$$
\begin{aligned}
& \gamma_{1}=\frac{1}{2}\left(\frac{1}{m_{L}}+\frac{1}{m_{H}}\right) \\
& \gamma_{2}=\frac{1}{2}\left(\frac{1}{m_{L}}-\frac{1}{m_{H}}\right)
\end{aligned}
$$

where $m_{\mathrm{L}}$ and $m_{\mathrm{H}}$ are the light hole and heavy hole effective masses. The band structure using k.p method is matched against the density functional theory (DFT) predicted values [18] in the region close to the valence band maximum at the $\Gamma$ point of the Brillouin zone by considering $m_{\mathrm{L}}$ and $m_{\mathrm{H}}$ equal to $0.071 m_{\circ}$ and $0.29 m_{\circ}$ respectively [18]. The $k . p$ calculations are in agreement with DFT results for a range of $\sim 0.2 \mathrm{eV}$, as can be seen in Fig. 2. It should be noted that since the Fermi level in our simulated p-MOSFET structure is below the valence band maximum level by $0.03 \mathrm{eV}$, the contributions from two valence bands can be readily captured by the proposed framework.

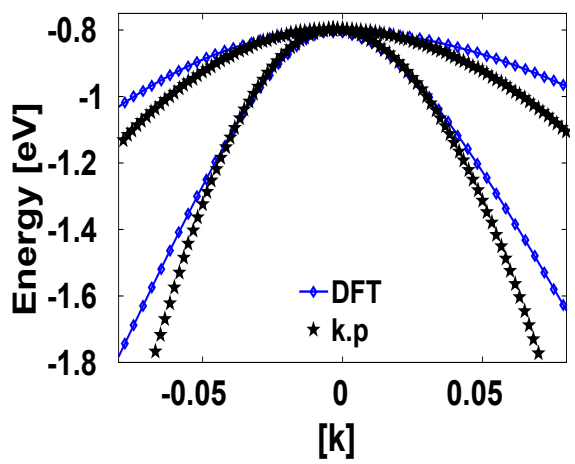

Fig. 2: Band-structure close to the $\Gamma$ point of Germanane chair valence band maximum. The 2-band k.p Hamiltonian shows a close agreement with the DFT calculations.
Since the main focus of this work is to meet the high performance on current limit, it is necessary to select an optimum source/drain doping for each length of the device. The source/drain doping for n-MOSFET and p-MOSFET with different channel lengths, has been chosen in the range varying from $3 \times 10^{12} / \mathrm{cm}^{2}$ to $5 \times 10^{12} / \mathrm{cm}^{2}$, in order to ensure that maximum $I_{\mathrm{ON}}$ is achieved for given $I_{\mathrm{OFF}}$ as per the ITRS HP requirements. It has been found ( not shown here) that doping lower than $10^{12} / \mathrm{cm}^{2}$ results in source exhaustion [23], whereas higher orders of doping result in degraded swing with the current rising rather slowly and not reaching the desired value at $V_{\mathrm{D}}=0.6 \mathrm{~V}$. These doping concentrations result in the degeneracy of $\sim 0.03 \mathrm{eV}$ and $0.14 \mathrm{eV}$ in p-MOSFET and n-MOSFET respectively. The channel region is intrinsic.

To model the ballistic transport, we solve Schrödinger's equation by using NEGF formalism self-consistently with the 2D-Poisson equation in the cross section of the device shown in Fig. 1. The retarded Green's function in the matrix expression is calculated as [24], [25]

$$
G^{r}\left(k_{y}, E\right)=\left[\left(E+i \eta^{+}\right) I-H\left(k_{y}\right)-\Sigma_{S}-\Sigma_{D}\right]^{-1}
$$

where $k_{y}$ is the transverse wave vector, $H\left(k_{y}\right)$ is the Hamiltonian matrix discretized along the transport direction $x, I$ is the identity matrix and $\Sigma_{S} / \Sigma_{D}$ are self-energy matrices for the source/drain contacts. In this study, ballistic transport is assumed due to the relatively short channel lengths considered and hence no scattering self-energy is included in Eq.(3). We consider a nano-sheet of Germanane and establish Bloch periodic boundary conditions along the transverse direction $y$. Moreover by neglecting mode mixing due to a smooth potential we implement uncoupled mode space approach for calculating NEGF equations, eventually resulting in computational savings. From the retarded Greens Function $G^{r}\left(k_{y}, E\right)$ we calculate the electron and hole Greens Function $G^{n}\left(k_{y}, E\right)$ and $G^{p}\left(k_{y}, E\right)$ as follows:

$$
\begin{aligned}
& G^{n}\left(k_{y}, E\right)=G^{r}\left(k_{y}, E\right) \Sigma_{n} G^{r \dagger}\left(k_{y}, E\right) \\
& G^{p}\left(k_{y}, E\right)=G^{r}\left(k_{y}, E\right) \Sigma_{p} G^{r \dagger}\left(k_{y}, E\right)
\end{aligned}
$$

where ,

$$
\begin{gathered}
\Sigma_{n} \equiv\left(\Sigma_{S / D}-\Sigma_{S / D^{\dagger}}\right) * f_{S / D} \\
\Sigma_{p} \equiv\left(\Sigma_{S / D}-\Sigma_{S / D}\right) *\left(1-f_{S / D}\right)
\end{gathered}
$$

with $\dagger$ denoting the transverse conjugate and $f_{S / D}$ denoting the Fermi-distribution function of source/drain. The carrier densities are calculated from the $j$ th diagonal elements of $G^{n}\left(k_{y}, E\right)$ and $G^{p}\left(k_{y}, E\right)$ as

$$
\begin{aligned}
& n_{j, j}=\sum_{k_{y}} \frac{1}{S} \int_{-\infty}^{+\infty} \frac{G^{n}\left(k_{y}, E\right)}{2 \pi} d(E) \\
& p_{j, j}=\sum_{k_{y}} \frac{1}{S} \int_{-\infty}^{+\infty} \frac{G^{p}\left(k_{y}, E\right)}{2 \pi} d(E)
\end{aligned}
$$

and the electron current density flowing from position $j$ to $j+1$ along the $x$ direction is calculated from the off-diagonal 
elements $(j, j+1)$ of $G^{n}\left(k_{y}, E\right)$ as

$$
\begin{gathered}
J^{n}{ }_{j, j+1}=\sum_{k_{y}} \frac{2 q}{\hbar S} \int_{-\infty}^{+\infty} \frac{d(E)}{2 \pi}\left[H_{j, j+1} G^{n}{ }_{j+1, j}\left(k_{y}, E\right)-\right. \\
\left.H_{j+1, j} G^{n}{ }_{j, j+1}\left(k_{y}, E\right)\right]
\end{gathered}
$$

where $S$ is the device cross-section along the transport direction. Finite difference method has been used to discretize the 2D Poisson's equation in the cross-section of the device with the inclusion of the Dirichlet boundary condition at the metal gate electrodes and the Neumann boundary condition at the left, right, top and bottom edges. The Poisson's equation solved is:

$$
\nabla^{2} U(z, x)=-\frac{\rho}{\epsilon}
$$

where $\mathrm{U}$ is the electrostatic potential, $\epsilon$ is the dielectric constant and $\rho$ is the charge density computed by the Recursive Green's Function Algorithm [26], [27]. The NEGF transport equation and the Poisson equation are solved iteratively until self-consistency is achieved. Mixed $\mathrm{C}$ and MATLAB $^{\circledR}$ [28] programming was implemented to speed up the Recursive Green's Function algorithm [29]. Parallel computation for the transverse modes carried out on a 3.6 $\mathrm{GHz}, 6$ core, CentOS workstation resulted in further reduction of the computational time.

\section{RESULTS AND DisCUSSIONS}

We first investigate the static current voltage characteristics of monolayer Germanane FETs followed by dynamic performance metrics. Transfer characteristics of both nMOSFET and p-MOSFET have been examined and the simulated $I_{\mathrm{D}^{-}} V_{\mathrm{G}}$ plots of p-MOSFET and n-MOSFET for varying channel lengths are shown in Fig. 3. The $I_{\mathrm{ON}}$ in monolayer Germanane n-MOSFET for $L_{\mathrm{CH}}=7 \mathrm{~nm}, 5 \mathrm{~nm}$ and $3 \mathrm{~nm}$ are $888.4 \mu \mathrm{A} / \mu \mathrm{m}, 1.7 \mathrm{~mA} / \mu \mathrm{m}$ and $2.4 \mathrm{~mA} / \mu \mathrm{m}$ respectively, whereas that for p-MOSFET are $697 \mathrm{nA} / \mu \mathrm{m}$, $1.44 \mathrm{~mA} / \mu \mathrm{m}$ and $4.16 \mathrm{~mA} / \mu \mathrm{m}$ respectively as shown in Table I. Therefore it is quite evident that devices with channel length scaled upto $3 \mathrm{~nm}$ show comparable ON-current as that of ITRS 2028 HP metric.

However, closer observation of the plots reveal an interesting trend. As the channel length increases the p-MOSFET starts performing better and exceeds the current in n-MOSFET. This phenomenon can be explained after studying the energy resolved current density spectrum plots in Fig. 4 to Fig. 6. The current spectrum plots for all the channel lengths of p-MOSFET and n-MOSFET have been calculated at that bias voltage where we see the current in p-MOSFET starting to cross over the current in n-MOSFET which is $V_{\mathrm{G}}=0.4 \mathrm{~V}$, $V_{\mathrm{D}}=0.6 \mathrm{~V}$. We also calculate the percentage of tunneling current $I_{\text {TUNNELING }}$ and current over the barrier $I_{\text {THERMIONIC }}$ for each of the channel lengths in both n-MOSFET and p-MOSFET at the said bias point as shown in Table II.

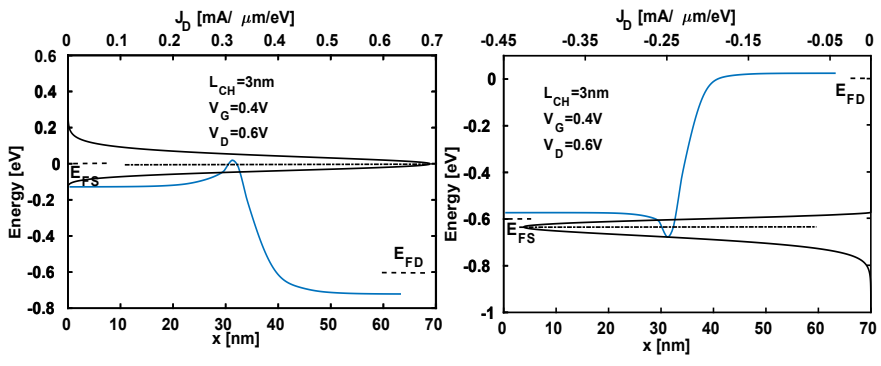

Fig. 4: Energy resolved current spectrum superimposed on the conduction band profiles for both (a) Germanane n-MOSFET and (b) p-MOSFET with channel length $3 \mathrm{~nm}$ (a)

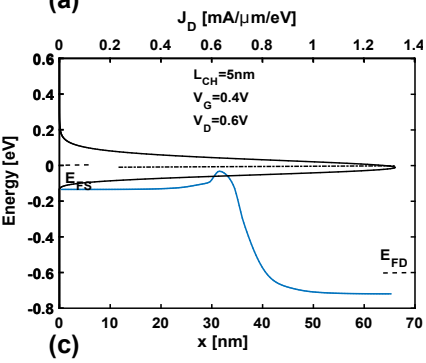

(b)

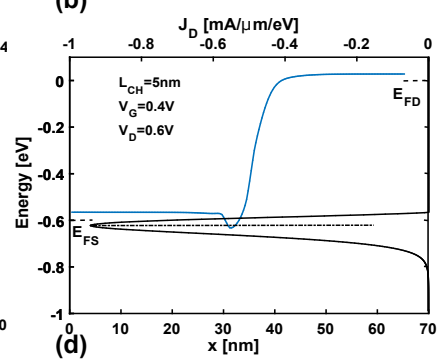

Fig. 5: Energy resolved current spectrum superimposed on the conduction band profiles for both (a) Germanane n-MOSFET and (b) p-MOSFET with channel length $5 \mathrm{~nm}$
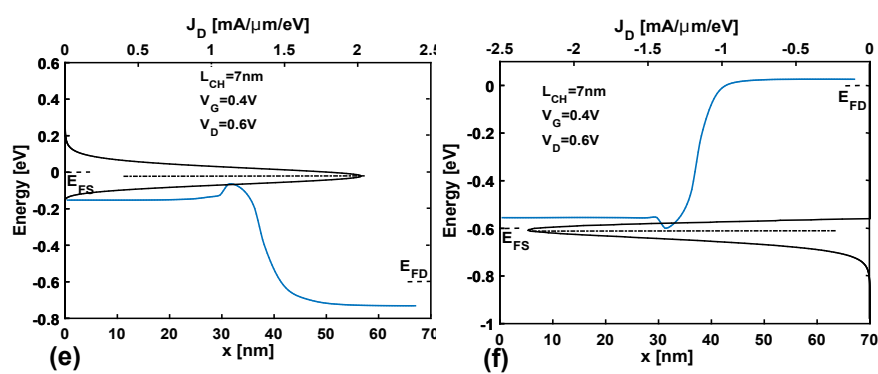

Fig. 6: Energy resolved current spectrum superimposed on the conduction band profiles for both (a) Germanane n-MOSFET and (b) p-MOSFET with channel length $7 \mathrm{~nm}$

We find that in shorter channel length devices the percentage of tunneling is more than the percentage of thermionic emission, thereby resulting in degraded slope of $I_{\mathrm{D}}-V_{\mathrm{G}}$ curves. As the channel length increases, the percentage of tunneling reduces in both $\mathrm{n}$ and $\mathrm{p}-\mathrm{MOSFET}$, attributing to improvement in the slope of the transfer characteristics. However, we see that the percentage reduction in tunneling in p-MOSFET from $5 \mathrm{~nm}$ to $7 \mathrm{~nm}$ is more pronounced that in case of n-MOSFET. Due to this the improvement of slope from shorter to higher channel lengths, is better in p-MOSFET than its n-counterpart and hence the on-current starts to exceed at higher gate overdrives. Further, the modulation of barrier height in both the devices play an important role in determining the current cross-over phenomenon. Studying Fig. 4 to 6 we find that, in shorter channel length devices the height of the barrier is 

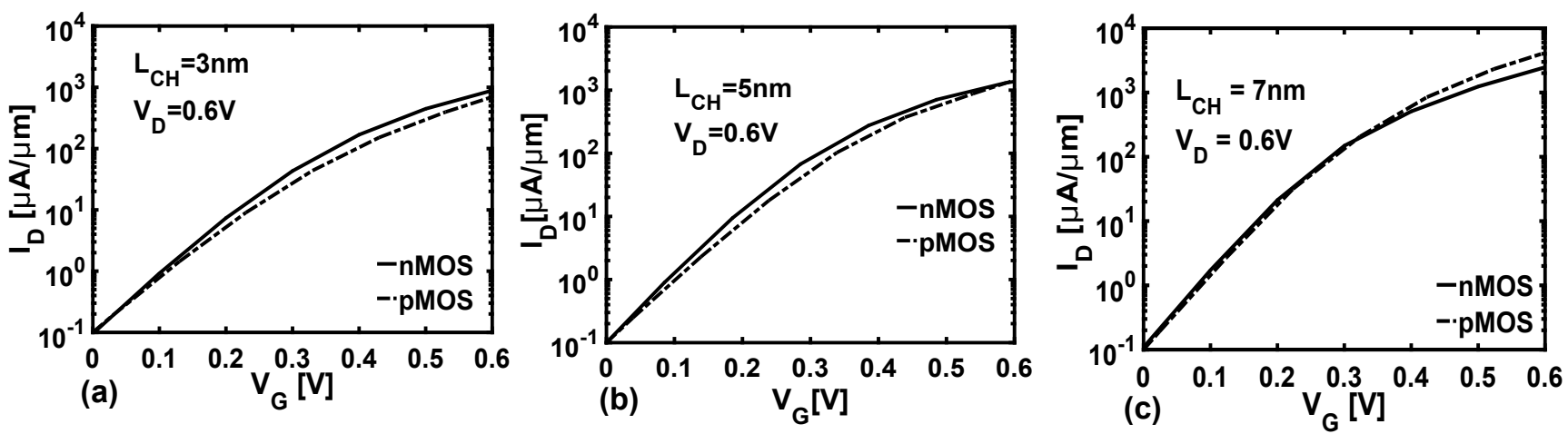

Fig. 3: Transfer characteristics of GermananeFETs for (a) $L_{\mathrm{CH}}=3 \mathrm{~nm}$ (b) $L_{\mathrm{CH}}=5 \mathrm{~nm}$ and (c) $L_{\mathrm{CH}}=7 \mathrm{~nm}$ and for a drain bias of $V_{\mathrm{D}}=0.6 \mathrm{~V}$.

such that the current is mostly tunneling. Therefore, the nMOSFET which has a lower effective mass results in a higher current value than the p-MOSFET. But, as the channel length increases, say for example $7 \mathrm{~nm}$, when the barrier height is very low in both the devices at high gate overdrives, the current is mostly due to thermionic emission. Here, the p-MOSFET having a higher effective mass attributing to higher denisty of states has more number of modes to fill and hence exhibits a higher current than n-MOSFET.

Next, the dynamic HP parameters like intrinsic switching delay $(\tau)$, total Gate to ground capacitance $\left(C_{\mathrm{G}}\right)$ and Power Delay Product (PDP) are calculated for all the channel lengths of n-MOSFET and p-MOSFET and compared. The values are shown in Table I. The intrinsic device delay, is computed following the method explained in [30] as $\tau=\left(Q_{\mathrm{ON}}\right.$ $\left.-Q_{\mathrm{OFF}}\right) / I_{\mathrm{ON}}$,where $Q_{\mathrm{ON}}$ and $Q_{\mathrm{OFF}}$ are the overall charges induced in the device at the ON-state and OFF-state, respectively. Total Gate to ground capacitance has been calculated by differentiating the total charge along the entire length of the device with respect to gate voltage as $C_{\mathrm{G}}=\delta Q / \delta V_{\mathrm{GS}}$. The PDP is calculated as $\mathrm{V}_{D D}\left(Q_{\mathrm{ON}}-Q_{\mathrm{OFF}}\right)$ [31]. In Fig. 7 we show the $C_{\mathrm{G}}$ values with respect to gate bias in case of both n- and p-MOSFETS. It is seen that in all cases $C_{\mathrm{G}}$ is higher in case of p-MOSFET, the reason being that it has a higher density of states than n-MOSFET and hence a larger quantum capacitance translating to a higher $C_{\mathrm{G}}$. The ultrascaled $\mathrm{n}$ - and p-MOSFETs where the transport is mainly due to source to drain tunneling, do not exhibit much difference in the magnitudes of $C_{\mathrm{G}}$. However, as the channel length increases and the devices entire the regime of higher gate overdrives $\left(V_{\mathrm{D}} \sim=0.3 \mathrm{~V}\right.$ to $0.4 \mathrm{~V}$ ), there is a significant change in the magnitude of the $C_{\mathrm{G}}$ values in case of ptype Germanane FETs. The reason is that, at such high gate overdrives, the barrier is very low in case of both $\mathrm{n}$ - and p-type FETs resulting mostly in thermionic emission, the p-MOSFET having higher density of states uncovers more number of modes thereby resulting in more modulation of charge with increment of the gate bias. The intrinsic switching delay, which is a manifestation of the gate capacitance, exhibits lower values in case of all the channel lengths of n-MOSFET than p-MOSFET. However, intrinsic switching delays for both p-
TABLE I: Performance Metrics comparison of Germanane Monolayer FETs with ITRS 2028 Prediction

\begin{tabular}{|c|rrr|rrr|r|}
\hline \hline Parameter & \multicolumn{2}{|c|}{ Germanane nMOS } & \multicolumn{2}{|c|}{ Germanane pMOS } & 2028 \\
& & & & & \\
& HP \\
\hline$L_{\mathrm{CH}}(\mathrm{nm})$ & 3 & 5 & 7 & 3 & 5 & 7 & 4.1 \\
\hline$I_{\mathrm{ON}}(\mathrm{mA} / \mu \mathrm{m})$ & 0.89 & 1.37 & 2.47 & 0.70 & 1.44 & 4.16 & 0.90 \\
\hline$\frac{I_{\mathrm{ON}}\left(\times 10^{3}\right)}{I_{\mathrm{OFFF}}}$ & 8.90 & 13.70 & 25 & 7.00 & 14.40 & 41.6 & 9 \\
\hline$C_{\mathrm{G}}(\mathrm{fF} / \mu \mathrm{m})$ & 0.12 & 0.16 & 0.22 & 0.14 & 0.51 & 0.90 & 0.60 \\
\hline$\tau(\mathrm{fs})$ & 72 & 57 & 39 & 107 & 69 & 47 & 423 \\
\hline$P D P(\mathrm{aJ} / \mu \mathrm{m})$ & 38 & 47 & 58 & 45 & 82 & 118 & 600 \\
\hline
\end{tabular}

TABLE II: Percentage of tunneling component and thermionic component of the total current in Germanane Monolayer FETs for all channel lengths at $V_{\mathrm{G}}=0.4 \mathrm{~V}, V_{\mathrm{D}}=0.6 \mathrm{~V}$

\begin{tabular}{|c|rrr|rrr|}
\hline \hline Parameter & \multicolumn{3}{|c|}{ Germanane nMOS } & \multicolumn{3}{|c|}{ Germanane pMOS } \\
\hline$L_{\mathrm{CH}}(\mathrm{nm})$ & 3 & 5 & 7 & 3 & 5 & 7 \\
\hline$I_{\text {TUNNELING }}(\%)$ & 60.80 & 27.93 & 14.30 & 76.89 & 49.68 & 28.15 \\
\hline$I_{\text {THERMIONIC }}(\%)$ & 37.96 & 72.70 & 85.70 & 23.11 & 50.32 & 71.85 \\
\hline
\end{tabular}

and n-MOSFETs are comparable to the ITRS 2028 values. It should be noted, that the values of intrinsic switching delay in this sub-deca nanometer Germanane MOSFETS, tend to be increasing with the scaling of the channel length. The reason is that, though the difference in magnitudes of $Q_{\mathrm{ON}}$ and $Q_{\mathrm{OFF}}$ decreases as manifested by the reduction in values of $C_{\mathrm{G}}$, the on-current value also decreases, and hence the overall ratio of $\left(Q_{\mathrm{ON}}-Q_{\mathrm{OFF}}\right)$ to $I_{\mathrm{ON}}$ increases thereby translating to an increment in the values of $\tau$.

Next, we compare the energy efficiency and the switching capability of Germanane monolayer FETs with respect to other 2D material FETs. 

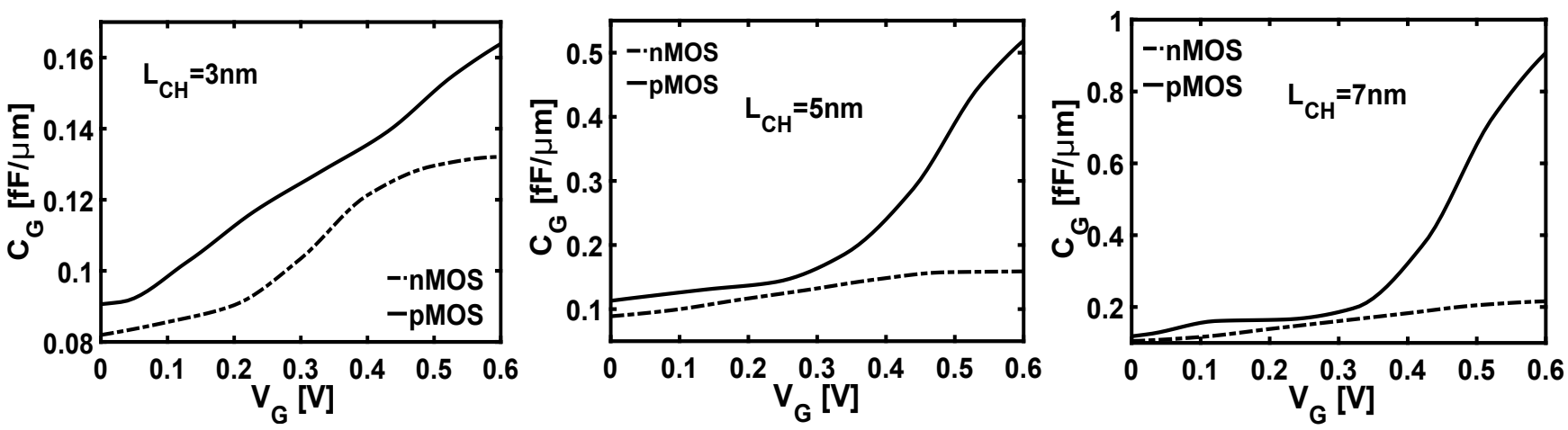

Fig. 7: Gate capacitance as a function of gate bias for (a) $L_{\mathrm{CH}}=3 \mathrm{~nm}, \mathrm{nMOSFET}$ and p-MOSFET, (b) $L_{\mathrm{CH}}=5 \mathrm{~nm}$, nMOSFET and p-MOSFET and (c) $L_{\mathrm{CH}}=7 \mathrm{~nm}, \mathrm{n}$-MOSFET and p-MOSFET.

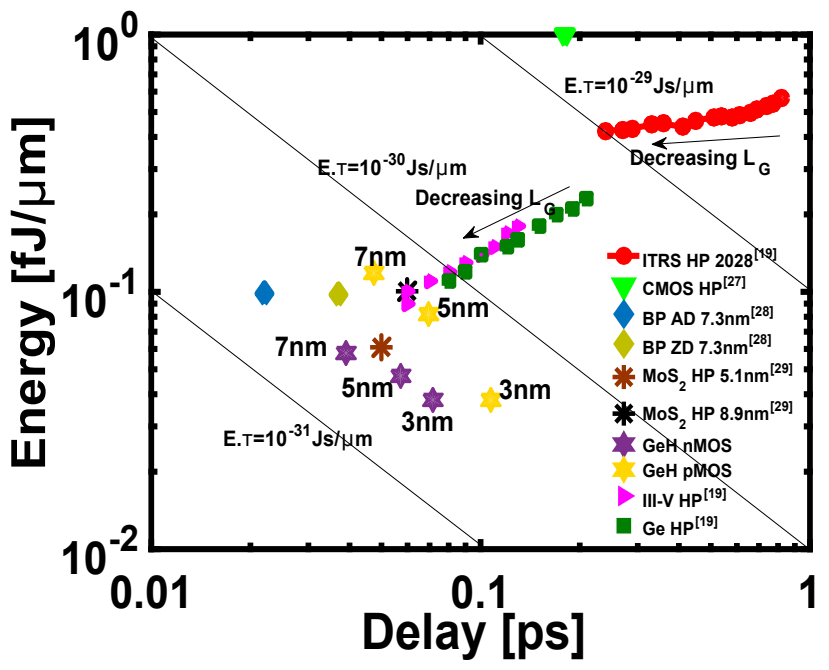

Fig. 8: Benchmarking the energy Vs delay with respect to the state of the art 2D materials and projected ITRS limits. E.T denotes the energy delay product.

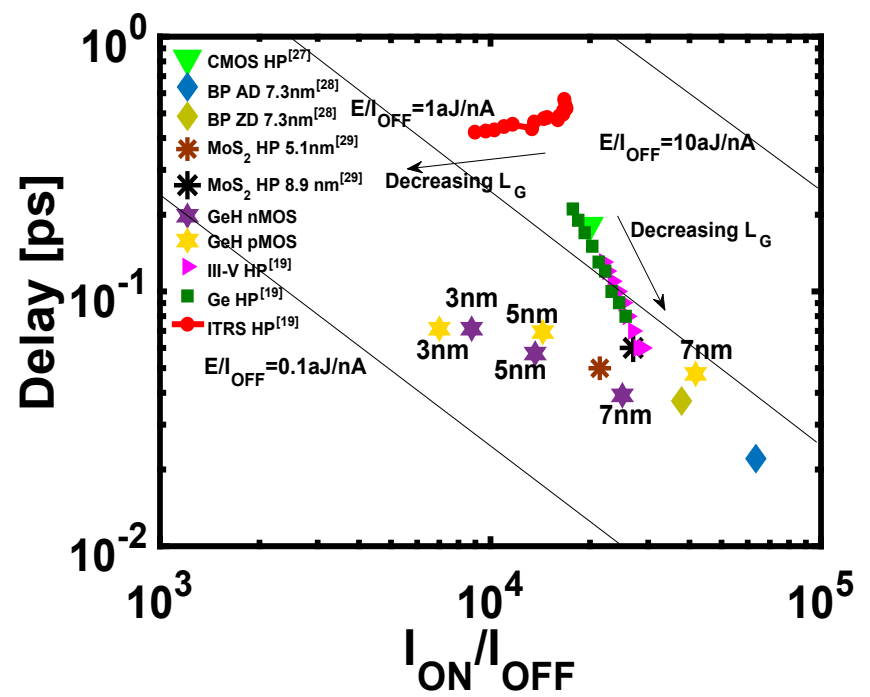

Fig. 9: Benchmarking the delay Vs $I_{\mathrm{ON}} / I_{\mathrm{OFF}}$ with respect to the state of the art $2 \mathrm{D}$ materials and projected ITRS limits.
In Figs. 8 and 9 we have plotted the switching energy versus the gate delay, and gate-delay versus $I_{\mathrm{ON}} / I_{\mathrm{OFF}}$ of these devices respectively. In the plots, CMOS HP stands for $15 \mathrm{~nm}$ technology node CMOS HP at $0.73 \mathrm{~V}$ [32]. BP AD and ZD stand for HP monolayer Black Phosphorus FETS in Armchair and Zigzag directions resp.,where the channel length is $7.3 \mathrm{~nm}$ with $V_{\mathrm{D}}=0.69 \mathrm{~V}$ [33]. Further, $\mathrm{MoS}_{2} \mathrm{HP}$ FETs for two different channel lengths are shown in the charts [34]. The ITRS predicted trend lines till the end of the roadmap, as well as III-V/Ge HP technologies are also shown in the plots for reference. The straight lines in Fig. 8 and Fig. 9 denote constant energy-delay products and constant ratios of switching energy to $I_{\mathrm{OFF}}$ resp. In both the charts, it can been seen that Germanane FETs meet the ITRS HP as well as III-V/Ge HP FET requirements very well. From Fig. 8 it is seen that the ultra scaled Germanane n-MOSFET has faster operation with a lower energy consumption with respect to the other 2D FETs. $7 \mathrm{~nm}$ Germanane n-MOSFET has almost similar performance as BP ZD FET, with lower energy consumption per switching event. On the other hand, Germanane p-MOSFETs has greater switching energy than their n-counterparts, but show comparable performance with respect to other 2D FETs. In Fig. 9 we see that the performance is best at higher channel lengths for both n- and p- Germanane FETs. Though they are not able to outperform the BP FETs in terms of faster switching and higher $I_{\mathrm{ON}} / I_{\mathrm{OFF}}$ ratios, their performance are better compared to the $\mathrm{MoS}_{2} \mathrm{HP}$ FETs. Also, CMOS HP has almost the same $I_{\mathrm{ON}} / I_{\mathrm{OFF}}$ ratio as the $7 \mathrm{~nm}$ Germanane FETs but at the cost of gate delay higher than an order of magnitude. Therefore as a whole, ultra-scaled Germanane FETs deliver a faster response at low switching energies, almost consistent energy-delay product, and practical $I_{\mathrm{ON}} / I_{\mathrm{OFF}}$ ratios with uniform $E / I_{\mathrm{OFF}}$ ratio -all of which combine to deliver a more energy efficient computation with a smaller footprint.

\section{CONCLUSION}

The high performance characteristics of Germanane FETs in the sub-deca nanometer regime seem to be well comparable to the ITRS 2028 predicted values. Both Germanane n-MOSFET 
and p-MOSFET offer excellent $I_{\mathrm{ON}}$ with large $I_{\mathrm{ON}} / I_{\mathrm{OFF}}$ ratios even when scaled down to $3 \mathrm{~nm}$ channel length, which is beyond the ITRS projected limits. Dynamic parameters such as total capacitance, intrinsic switching delay and switching energy have also been calculated ans we show that n-MOSFET performs better in comparison to the p-MOSFET, though both the devices meet the ITRS projected limits. Our study therefore suggests that Germanane FETs possess benefits for high-performance device applications even in the sub-deca nm regime.

\section{REFERENCES}

[1] B. Radisavljevic, M. B. Whitwick, and A. Kis, "Integrated circuits and logic operations based on single-layer $\mathrm{MoS}_{2}$," ACS nano, vol. 5, no. 12, pp. 9934-8, Dec. 2011.

[2] H. Fang, S. Chuang, T. C. Chang, K. Takei, T. Takahashi, and A. Javey,"High-performance single layered WSe 2 p-FETs with chemically doped contacts," Nano Lett., vol. 12, no. 7, pp. 3788-3792, Jun. 2012.

[3] L. Liu, S. Bala Kumar, Y. Ouyang, and J. Guo, "Performance limits of monolayer transition metal dichalcogenide transistors," IEEE Trans. Elec. Dev., vol. 58, no. 9, pp. 3042-3047, Sep. 2011

[4] R. K. Ghosh and S. Mahapatra, "Monolayer transition metal dichalcogenide channel based tunnel transistor," IEEE J. Elec. Dev. Soc., vol. 1, no. 10, pp. 175-180, Oct. 2013.

[5] Likai Li, Yijun Yu, Guo Jun Ye, Qingqin Ge, Xuedong Ou, Hua Wu, Donglai Feng, Xian Hui Chen and Yuanbo Zhang, "Black Phosphorus Field Effect Transistors," Nature Nanotech., vol. 9, no. 5, pp. 372-377, May. 2014

[6] Han Liu, Adam T. Neal, Zhen Zhu, Zhe Luo, Xianfen Xu, David Tomnek, Peide D. Ye, "Phosphorene: An Unexplored $2 D$ Semiconductor with a High Hole Mobility," ACS Nano., vol. 8, no. 4, pp. 4033-4041, March 2014.

[7] D. Golberg et al., "Boron nitride nanotubes and nanosheets," ACS Nano, vol. 4, no. 6, pp. 2979-2993, May 2010.

[8] K. S. Novoselov et al., "Two-dimensional atomic crystals," PNAS, vol 102, no. 30, pp. 10451-10453, Jul. 2005.

[9] KJ Koski, Y Cui, “ The new skinny in two-dimensional nanomaterials,"ACS Nano, vol. 7, no. 5, pp 3739-3743, May 2013.

[10] Zakaria Y. Al Balushi et. al "Two-dimensional gallium nitride realized via graphene encapsulation," Nature Materials, vol. 15, pp. 1166-1171, August 2016.

[11] L. C. Lew Yan Voon, E. Sandberg, R. S. Aga, and A. A. Farajian, "Hydrogen compounds of group-IV nanosheets," Appl. Phys. Lett., vol. 97 , no. 16, September 2010.

[12] Joelson C. Garcia, Denille B. de Lima, Lucy V. C. Assali, and Joo F. Justo, "Group IV Graphene- and Graphane-Like Nanosheets," J. Phys. Chem. C, vol. 115, no. 27, pp 13242-13246, June 2011.

[13] O. Pulci, P. Gori, M. Marsili, V. Garbuio, R. Del Sole, and F. Bechstedt,"Strong excitons in novel two-dimensional crystals: Silicane and Germanane," Europhysics Lett., vol. 98, no. 3, May 2012.

[14] E. Bianco, S. Butler, S. Jiang, O. D. Restrepo, W. Windl, and J. E. Goldberger, "Stability and exfoliation of Germanane: A germanium graphane analogue," ACS Nano, vol. 7, no. 5, pp. 4414-4421, Mar. 2013

[15] Walid Amamou et al., "Large area epitaxial germanane for electronic devices." 2D Materials, vol. 2, no. 3, August, 2015

[16] B. N. Madhushanka et al., "Electronic properties of germanane fieldeffect transistors,"2D Materials, vol. 4, no. 2, February, 2017.

[17] Nanda Gopal Sahoo et al., "Schottky diodes from 2D germanane," Appl. Phys. Lett., vol. 97, no. 16, September 2010

[18] Ram Krishna Ghosh, Madhuchhanda Brahma and Santanu Mahapatra, "Germanane: A Low Effective Mass and High Bandgap 2-D Channel Material for Future FETs," IEEE Trans. Elec. Dev., vol. 61, no. 7, pp. 2309-2315, June 2014

[19] Low Kain Lu et al., "Ballistic Transport Performance of Silicane and Germanane Transistors,'IEEE J. Elec. Dev. Soc., vol. 61, no. 5, pp. 15901598, April 2014.

[20] Abdul Aziz AlMutairi et al., "Performance Limit Projection of Germanane Field-Effect Transistors," IEEE Elec. Dev. Lett., vol. 38, no. 5, pp. 673-676, May 2017.
[21] International Technology Roadmap for Semiconductors. [Online]. Available:http://www.itrs2.net/2013-itrs.html

[22] IV Tokatly, "Orbital momentum Hall effect in p-doped graphane," Phys. Rev. B, vol. 82, no. 16, October 2010.

[23] Raseong Kim ,Uygar E. Avci and Ian Young, "Source/drain doping effects and performance analysis of ballistic III-V n-MOSFETs," IEEE J. Elec. Dev. Soc., vol. 3, no. 1, pp. 37-43, October 2014.

[24] Supriyo Datta "Quantum transport: atom to transistor," Cambridge University Press, 2005.

[25] J. Guo, "Carbon nanotube electronics: Modeling, physics, and applications," Ph.D. dissertation, Dept. Elect. Eng., Purdue Univ., West Lafayette, IN, USA, 2004.

[26] R. Lake, G. Klimeck, R. Bowen, D. Jovanovic, "Single and multiband modeling of quantum electron transport through layered semiconductor devices," J. Appl. Phys. , vol. 81, no. 12, pp. 7845-7869 , February 1997.

[27] M. P. Anantram, Mark S. Lundstrom, and Dmitri E. Nikonov. "Modeling of Nanoscale Devices, " Proceedings of the IEEE, vol. 96, no. 9, pp. 1511-1550, Sept. 2008.

[28] MATLAB version 9.1.0.441655 (R2016b), September 7, 2016.

[29] Zhengping Jiang, "Quantum transport in RTD and atomistic modeling of nanostructures," Masters dissertation, Dept. Elect. Eng., Purdue Univ., West Lafayette, IN, USA, 2011.

[30] S. O. Koswatta, M. S. Lundstrom, and D. E. Nikonov, "Performance comparison between p-i-n tunneling transistors and conventional MOSFETs," IEEE Trans. Elec. Dev., vol. 56, no. 3, pp. 456-465, March 2009.

[31] D. Logoteta, G. Fiori and G. Iannaccone, "Graphene-based lateral heterostructure transistors exhibit better intrinsic performance than graphene-based vertical transistors as post-CMOS devices ," Scientific reports, vol. 4, October 2014.

[32] K. Majumdar, C. Hobbs, and Paul D. Kirsch, "Benchmarking Transition Metal Dichalcogenide MOSFET in the Ultimate Physical Scaling Limit," IEEE Elec. Dev. Lett., vol. 35, no. 3, pp. 402-404, Maech 2014.

[33] Fei Liu, Yijiao Wang, Xiaoyan Liu, Jian Wang, and Hong Guo, "Ballistic transport in monolayer black phosphorus transistors," IEEE Trans. Elec. Dev., vol. 61, no. 11, pp. 3871-3876, November 2014.

[34] Leitao Liu, Yang Lu, and Jing Guo, "On Monolayer MoS2 Field-Effect Transistors at the Scaling Limit," IEEE Trans. Elec. Dev., vol. 60, no. 12, pp. 4133-4139, December 2013. 